\title{
Method for PE Pipes Fusion Jointing Based on TRIZ Contradictions Theory
}

\author{
Jianguang Sun ${ }^{1}$, Runhua Tan ${ }^{1}$, Jinyong $\mathrm{Gao}^{2}$, and Zihui Wei ${ }^{1}$ \\ ${ }^{1}$ School of Mechanical Engineering, Hebei University of Technology, Tianjin, PRC \\ ${ }^{2}$ School of Information Engineering, Hebei University of Technology, Tianjin, PRC \\ \{ Jianguang. Sun, Runhua. Tan, Jinyong. Gao, \\ Zihui.Wei,sjg\}@hebut.edu.cn
}

\begin{abstract}
The core of the TRIZ theories is the contradiction detection and solution. TRIZ provided various methods for the contradiction solution, but all that is not systematized. Combined with the technique system conception, this paper summarizes an integration solution method for contradiction solution based on the TRIZ contradiction theory. According to the method, a flowchart of integration solution method for contradiction is given. As a casestudy, method of fusion jointing PE pipe is analysised.
\end{abstract}

Keywords: TRIZ, contradiction theory, physical contradiction, separation principle, fusion jointing.

\section{Introduction}

TRIZ is the Russian abbreviation of what can be translated as 'the theory of inventive problem solving' and is an integrative system info that is composed of varieties of methods and tools for solving technology problem and realizing innovation. The core of products innovation is to solve contradiction in the design[1], so the theory of contradiction solution is the core of TRIZ. Altshuller, the inventor of TRIZ, researches the methods of solve contradiction based on many famous inventor patents in the world, including inventive principles, standard solution, and separation of physical contradiction.

In recent years numerous scholars concentrate on how to detect contradiction[2], and adopt other theories to detect contradiction, such as QFD, TOC, AD, etc[3][4][5]. Then combining the TRIZ contradiction solution methods form the intact solve process of contradiction detect. But there are many problems to be solved in the exiting contradiction solve methods of TRIZ, a good analysis and solve contradiction method in the system is not be presented. Based on summarizing the concepts and methods relative to contradiction in TRIZ, applying the concept of TRIZ systematology and available resource, the paper proposes the analysis and solving method in technology system, and puts forward some improving and developing direction on the TRIZ contradiction theory. 


\section{Methodology}

\subsection{The Concept of TRIZ Systematology}

TRIZ is a systematized method. The systematology is the important thought of research problem in TRIZ. The system includes the meaning of the following aspects:

1. Physical structure of technology system: physical structure of technology system is an organic whole composed of several elements by certain structural form coupling that can realize some kind of function.

2. Operation process of technology system: Technology system consists of input raw material, tool, auxiliary tool, product, etc. the operation process of technology system can be described as the input raw material translating into product under the function of tool and auxiliary tool.

3. As a whole, the system is not isolation to act on the environment through the function of input and output.

The system is composed of the subsystem (or element). In a system, the subsystem has the relatively obvious boundary with the periphery part and has the complete structure and function, or is called the component elements. Subsystem itself also is a system, also may decompose downward, and decomposes continuously to the part and the operation up to.

The system is one part of the super-system. One kind of description about the system, the subsystem, and the super-system is shown in Fig.1.

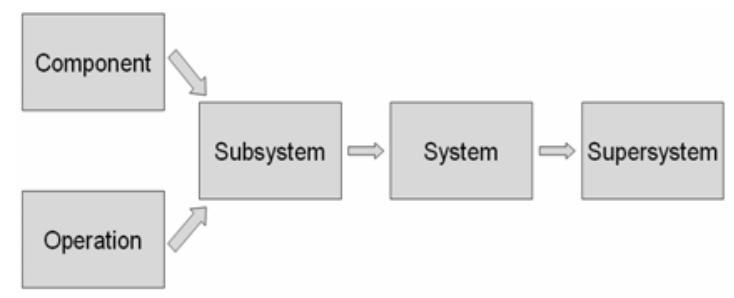

Fig. 1. The system structure diagram by TRIZ

Solving the contradiction in the system and between the systems should have the total view, should follow the following two principles:

1. Overall principle: the change in the system does not affect the entire super-system.

2. System minimum modification principle: as much as possible existing resources of the system are applied, as little as possible the new materials are introduced.

\subsection{Technical Contradiction}

TRIZ is mainly to solve technical contradiction and physical contradiction. Technical contradiction refers to one function simultaneously to cause useful and harmful two results, may also refer to the useful function introduced either the ill effect eliminated 
causes some performance of one system or several systems get worse. The technical contradiction often appears as the contradiction between two subsystems in a system, which is caused by both sides of contradictions.

Altshuller through in-depth study of the conflict has found 39 engineering parameters, a couple of them can available to describe every technical contradiction, also has found that every technical described by a couple parameters of them has innovation solution and can obtain it by the definitive method. These methods are concluded 40 innovation theories. Altshuller also has created a contradiction matrix, of which first line and first row elements are both formed by the 39 engineering parameters and other elements are given the serial number of the 40 innovation theories to solve relevant technical contradiction.

To solve technical contradiction by the contradiction matrix and the innovation theory is based on the experience and analogy thought innovation method. The innovation theories in the contradiction matrix are the similar historical success solutions of contradiction. The different contradiction's special condition is infinitely varied, and humanity's innovation is realizing breakthrough in the incessant, the innovation theory can not always solve the contradiction.

\subsection{Physical Contradiction and the Method for Solution}

The technical contradiction thorough analysis may transform into the physical contradiction, the physical contradiction is even more near to the question essence. In the product design, some part simultaneously displaying two opposite conditions is called the physical contradiction.

TRIZ has provided the following 4 separation principle to solve physical contradiction:

1. Separates the opposite characteristic from the time: In a time section the object shows one characteristic, in another time section object shows another characteristic.

2. Separates the opposite characteristic from the space: one part of the object is for one characteristic, another part of the object is for another characteristic.

3. Separates the opposite characteristic from the whole and the part: the whole has one characteristic, but the part has the opposite characteristic.

4. Opposite characteristic coexistence in the same substance: The substance displays the only characteristic under the specific condition and displays another under another kind of condition characteristic.

After a question is analyzed thoroughly, often the separation principle is first to be use for solving physics conflict. TRIZ will take discovering and solving physics contradiction as the judgment standard of a very good solution for the question.

\subsection{Su-Field Analysis and Standard Solution}

Altshuller's one of contributions to TRIZ is that he proposes the substance-field description method and model for the function. Its principle is, all functions may be decomposes into two substance and one kind of field, namely one kind of function is composed of two substance and one kind of field. 
The substance-field model may express the relations between elements. All systems are to realize some kind of function, the input and output elements correspond to two substances in the substance-field model, the field corresponds to tool element. The substance-field model is a description pattern by graphic that bases on the function expressing and realizing theory.

Based on this model, Altshuller proposes 76 kind of standard solutions, and divide into the following 5 kinds:

1. Does not change or the fewest change the existing system: 13 kind of standard solutions;

2. Changing the existing system: 23 kind of standard solutions;

3. System transferring: 6 kind of standard solutions;

4. Inspecting and measuring: 17 kind of standard solutions;

5. Reducing and improving strategy: 17 kind of standard solutions;

The standard solution mainly aims at the system's insufficiency and the contradiction improvement to perfect the system, not only is used in the contradiction resolution.

The 1st kind of standard solution under the condition of non-increasing the complex of the system takes the standard solution 1.1 (improve the non-complete function system, including 8 kind of solutions) to solve system's insufficiency, takes the standard solution 1.2 (eliminate or counter-balance ill effect, including 5 kind of solutions) to solve contradiction in the system.

The 2nd kind of standard solution is to improve the system through greater changing its substance-field model.

The 3rd kind of standard solution transmits the question in system into the supersystem and the subsystem, but changes does not limit in the system.

The 4th kind of standard solution is suitable for the system containing the examination or survey.

The function of 5th kind of standard solution is to reduce and improve the solution.

\subsection{Integration Method of Solution for Contradiction}

The existing contradiction solution tool of TRIZ takes contradiction as an isolated question to solve, has not considered the relation between the different elements in system. Through using the existing tool of TRIZ, unifying the systematology concept of TRIZ, deep-in analyzing the problem in process of solving contradiction in system, the following the integration method of solution for contradiction is presented.

1. System analysis: States the major function of system, the system's main elements include input material, tool, auxiliary tool and output product. If in the system lacks the tool module, then applies standard solution 1.1.

2. Determining contradiction region, contradiction time and system element concerning in contradiction. The contradiction in system occurs generally in tool element function process.

3. Constructing technology contradiction model, finding the improvement and worsened parameter in the system, using the contradiction matrix and the innovation theory to attempt to solve the technology contradiction. 
4. Applying system's available resource, and constructing physical contradiction. In turn selecting available resource to be improved, through the ideal solution discovering physical contradiction, and adopting the separation principle solve the physical contradiction, the concrete step as shown in Fig.2.

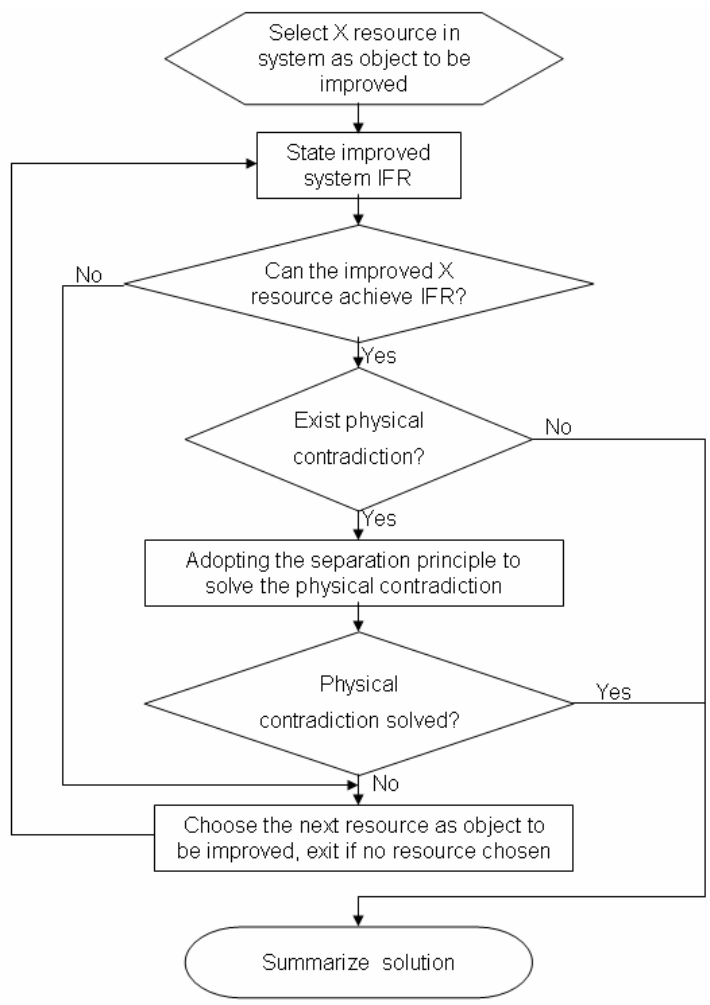

Fig. 2. The process of solving physical contradiction

5. Applying the substance-field analysis and the standard solution. Based on the system minimum modification principle, applies 1st, 2nd, and 3rd standard solutions in turn, and uses 4th standard solutions when involving the examination and the survey application.

6. Evaluating and optimizing for the solution program.

Considering that whether the solution program is the final solution, the contradiction solution of TRIZ can be divided into two points.

1. Discrete solution: the contradiction is thoroughly eliminated, or the new solution enables the original contradiction not to exist.

2. Continuous solution: The new solution eliminates the contradiction partly, but contradiction still exists. The contradiction is eliminated ceaselessly while a series of new contradiction generate, and these contradictions constitute the contradiction 
chain. Finally, the 5th kind of standard solution is applied to optimize the final solution. The flow chart of integration method of solution for contradiction is shown in Fig.3.

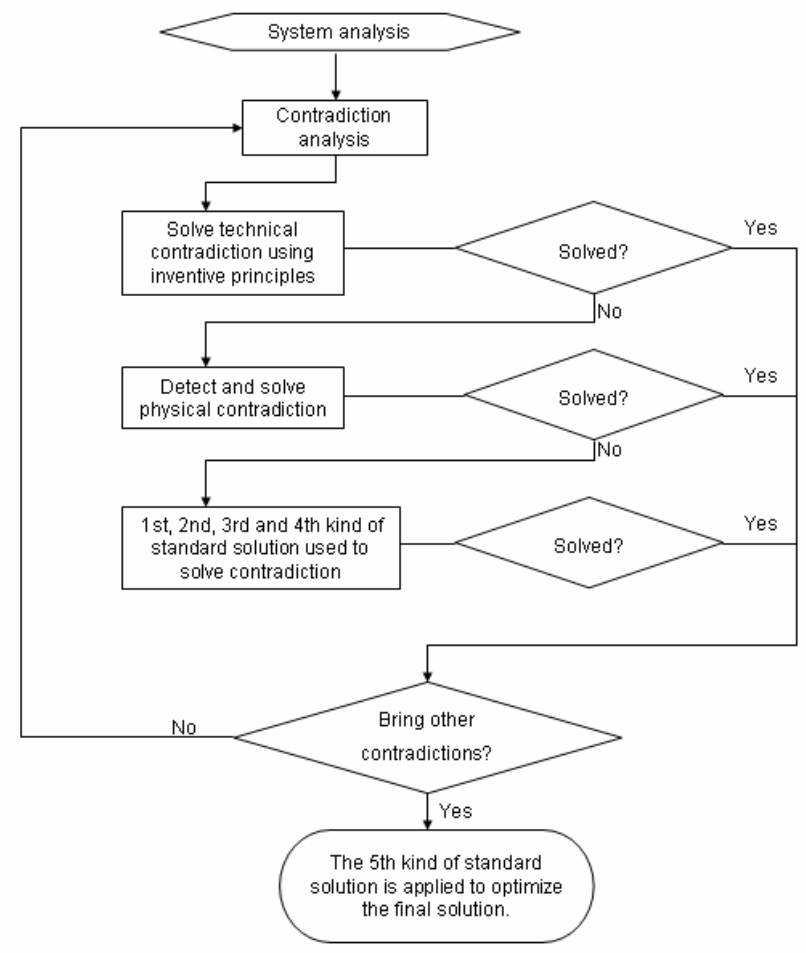

Fig. 3. The process of integration solution method for contradiction

TRIZ divides the solution of patent and question into 5 levels:

The 1st level: The conventional solution refers to has not solved any conflict. Usually, using the innovation theory may obtain the 2nd level solution.

The 2nd level: system change, namely the close or similar method for solving contradiction is used in solving the current system's contradiction.

The 3rd level: the cross profession's solution. The system's contradiction is solved in one branch of knowledge scope. Some elements may change essentially, and the 3rd level solution may be gained by using the separation principle and the standard solution.

The 4th level: the cross branch of knowledge solution. The system contradiction can be solved by the method of cross branch of knowledge. The 4th level solution needs the effect library of TRIZ.

The 5th level: finding, generally means the new effect appearance, even the new engineering project generation. The integration method of solution for contradiction proposed in the paper can maximumly obtain the 3rd level solution under the system minimum modification principle, which can solve the mostly problem in the fact. 


\section{Case Study-the Connection of Plastic Pipeline}

The plastic is a kind of high polymer material which is mainly used the petroleum or the coal as the raw material. Polyethylene(PE) Pipes, one of plastic's important application domains, have been widely used in the urban water supply and drainage, the buildings water supply and drainage, the hot water supply, heats supply, urban fuel gas, rain water draining water, urban fuel gas, agricultural irrigation and drainage, chemical fluid transportation as well as electric wire, cable sheath, etc[6]. The process of plastic welding is to make the plastic's contact surface to heat to the melted state, and then under the pressure function connects them together.

Usually selects the butt fusion joint method to connect the PE pipes. As shown in figure 1, firstly, both facing surfaces of pipes must be planed to smooth(Fig.4-A), then heats up the heating element. As soon as the heating element reaches set temperature, close the pipes by the hydraulic device with heating element in the middle(Fig.4-B). After set time elapsed, draws out the heating element immediately and butts joint the pipelines quickly(Fig.4-C), and then completes the pipe jointing process.

Contradiction appearance: In order to achieve the enough strength, the request of fusing area's cross-section is as far as possible big, but to achieve the ideal flow rate effect, requests the flanging fusing area cross-section to be as far as possible small, so this physical conflict must be solved.

Uses the spatial separation principle to solve this physical contradiction: As shown in Figure 5, the fusion region is two regions, namely, pipeline inside region $\mathrm{B}$ and pipeline outside region $\mathrm{C}$. The increase of region $\mathrm{B}$ will cause the pipeline flow rate to reduce, but the increase of region $\mathrm{C}$ will not affect to the pipeline flow rate. Therefore the spatial separation method is adopted. In order to achieve the enough strength the area of region $\mathrm{C}$ is increased, and to achieve the ideal flow rate effect, the region $\mathrm{B}$ area is reduced as far as possible, socket welding is selected(Fig.6), so the contradiction is solved.

In virtue of traditional butt fusion jointing is unable to realize the above separation heating effect, therefore the new jointing innovation theory should be sought. Due to the corresponding relationship of the separation principle and Altshuller's inventive principles[2], through searching the inventive principles, the principle 7- Nested structures is selected to be used, as shown in Figure 6. The socket welding method is adopted, which is equivalent to region $\mathrm{B}$ reduced to 0 and region $\mathrm{C}$ increased greatly, to realize ideal solution for the contradiction.

However, a new physical contradiction turns up as using of socket welding. In order to connect reliably, the end of plastic pipe is required to have special structure (large diameter for inserting easily) as shown in Fig.6, but the plastic pipe adopts the continuous extrusion when producing and the end is not allowed to be designed to this kind of structure. To solve the above-mentioned physical contradiction, the space separation principle is used to separate the socket from the pipe as shown in Fig.7.

Adopting the 3rd standard solution to design an electrofusion fitting. The structure of electrofusion fitting is shown in Fig.7. The connecting pipe is inserted into the the electrofusion fitting which is used as intermediary, After the resistance wire is energized, inner wall of fitting is heated and melted to achieve the objective of the welding. The electrofusion is a good method to solve the second physical contradiction. 

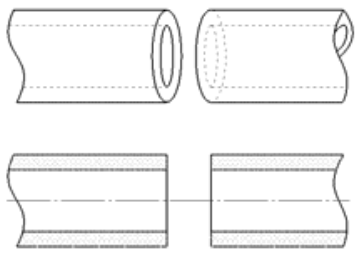

A

Precision hesting elenent, PTFE costed
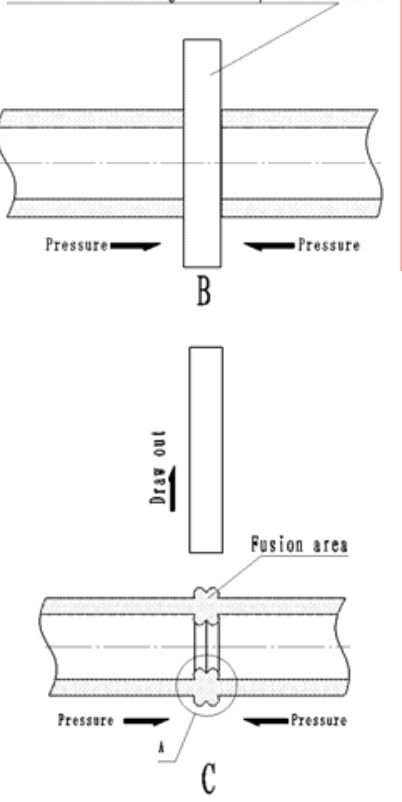

Fig. 4. The process of butt fusion for plastic pipes
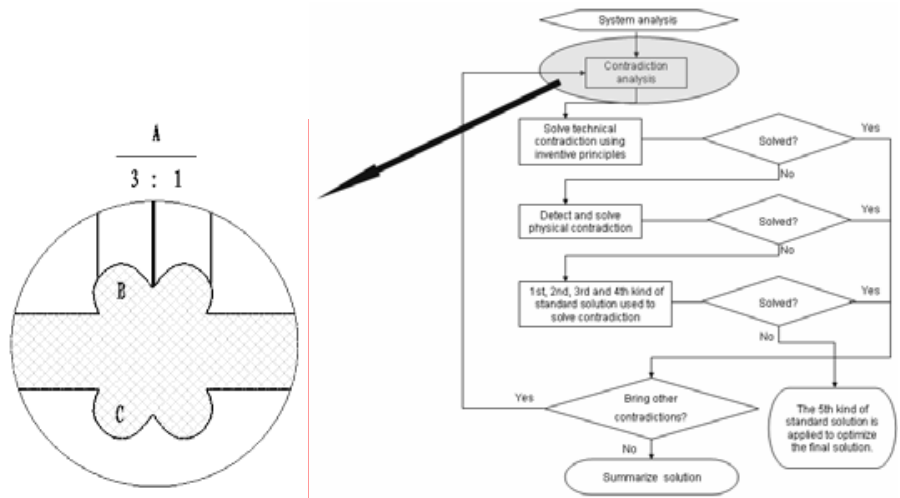

Fig. 5. Analysis of the first physical contradiction 


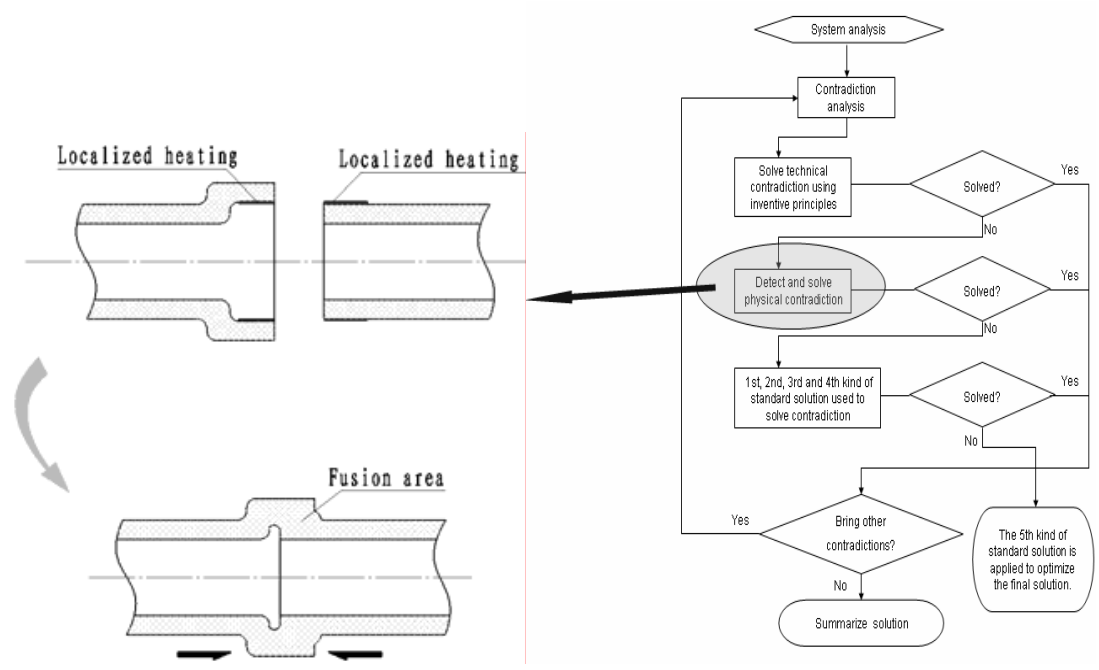

Fig. 6. The socket welding method is adopted according to separation principle

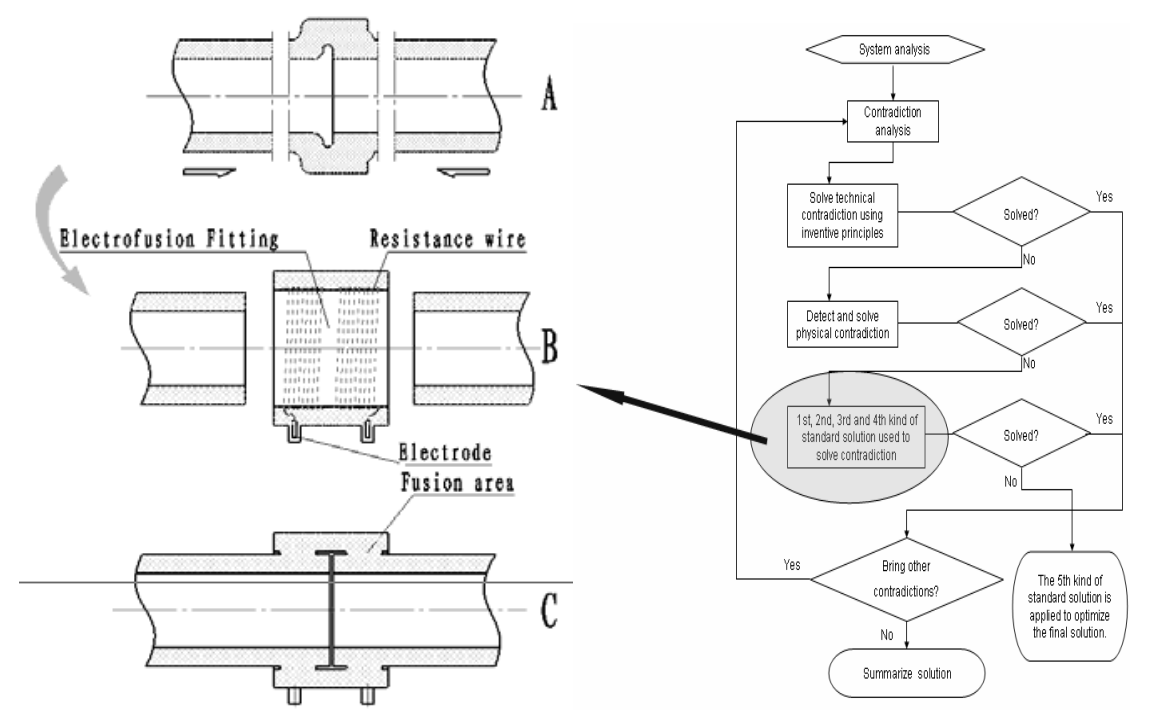

Fig. 7. A new physical contradiction appears and the second physical contradiction has been solved by electrofusion method

\section{Conclusion}

On the basis of the existing contradiction theory and solution method, the paper summarizes a integration solution method for contradiction. Through the adoption of this method, 40 inventive principles, separation principle and 5 standard solutions are 
effectively applied to solve a contradiction. Through analyzing on the method of fusion jointing plastics pipe, two physical contradicions is found. According to the integration solution method, all the contradiction is solved.

\section{Acknowledgment}

This research is supported in part by the Natural Science Foundation of Hebei under Grant Numbers E2008000101, the Key Project of the Ministry of Science and Technology of the People's Republic of China under Grant Numbers 2008IM030100, and the science and technology key project of Hebei Province under Grant Numbers 09212102D. Any opinions or findings of this work are the responsibility of the authors, and do not necessarily reflect the views of the sponsors or collaborators.

\section{References}

1. Altshuller, G.: The Innovation Algorithm, TRIZ, systematic innovation and technical creativity. Technical Innovation center, Worcester (1999)

2. Savransky, S.D.: Engineering of Creativity. CSC Press, New York (2000)

3. Cavallucci, D.: Integrating altshuller's development laws for technical systems into the design process. Annals of the CIRP 5(1), 115-120 (2001)

4. Noel, L.: A new model of the conceptual desig nproces susing QFD /FA/TRIZ. TRIZ Journal, http: / / www.triz-journal.com

5. Terninko, J.: Selecting the best direction to create the ideal product design. TRIZ Journal, http: //www.triz-journal.com

6. Xun, S.: Development of Polyethylene Pipes for Gas Burning in 90's. Chemical Materials for Construction 4, 9-12 (2000) 